\title{
Conversion to monotherapy with luteinizing-hormone releasing hormone agonist or orchiectomy after reaching PSA nadir following maximal androgen blockade is able to prolong progression-free survival in patients with metastatic prostate cancer: A propensity score matching analysis
}

\author{
GYEONG EUN MIN ${ }^{1}$ and HANJONG AHN ${ }^{2}$ \\ ${ }^{1}$ Department of Urology, Kyung Hee University College of Medicine, Seoul 05278; ${ }^{2}$ Department of Urology, \\ University of Ulsan College of Medicine, Asan Medical Center, Seoul 05505, Republic of Korea
}

Received July 27, 2015; Accepted January 10, 2017

DOI: $10.3892 / 01.2017 .6056$

\begin{abstract}
The present study evaluated androgen deprivation methods to determine the approach that most improves the progression-free survival (PFS) of patients with metastatic prostate cancer. Patients had received continuous maximal androgen blockade (MAB) or monotherapy [luteinizing-hormone releasing hormone (LHRH) agonist or orchiectomy] following the reaching of the prostate specific antigen (PSA) nadir. The medical records of 293 patients who received $\mathrm{MAB}$ following a diagnosis of metastatic prostate cancer were retrospectively reviewed. Following attainment of the PSA nadir and treatment with MAB, patients were maintained on continuous MAB (group CMAB) or converted to monotherapy (group MONO). Disease progression, defined as progression to castration-resistant prostate cancer, was evaluated and compared between the treatment modalities. PFS was compared between patients who received CMAB vs. MONO using 2:1 (102:53) propensity score matching; the basic clinicopathological characteristics (age, Gleason score, PSA and extent of bone metastasis) were similar between the groups. Disease progression was observed in $70.9 \%$ of all patients, with a median treatment period of 22.7 months. The median PFS time was 19.5 months in the CMAB group and
\end{abstract}

Correspondence to: Professor Hanjong Ahn, Department of Urology, University of Ulsan College of Medicine, Asan Medical Center, 88 Olympic-Ro 43-gil, Songpa-gu, Seoul 05505, Republic of Korea

E-mail: hjahn@amc.seoul.kr

Abbreviations: ADT, androgen deprivation therapy; PFS, progression-free survival; MAB, maximal androgen blockade; LHRH, luteinizing-hormone releasing hormone; PSA, prostate specific antigen; CRPC, castration-resistant prostate cancer

Key words: prostatic neoplasm, androgen, disease-free survival, neoplasm metastasis
28.8 months in the MONO group $(\mathrm{P}=0.008)$. Kaplan-Meier analysis demonstrated that PFS was significantly associated with the type of maintenance androgen deprivation therapy (ADT; log rank <0.005). Multivariate analysis revealed that the type of maintenance ADT and the pretreatment extent of bone metastasis were independent predictors of prolonged PFS. In this propensity score matched-analysis, conversion to monotherapy with a LHRH agonist or orchiectomy following attainment of the PSA nadir with initial MAB, prolonged the PFS, suggesting that monotherapy maintenance following initial MAB may benefit patients by reducing side effects without decreasing treatment efficacy.

\section{Introduction}

Prostate cancer is the most prevalent malignancy in men and the second most frequent cause of cancer-associated mortality in the United States (1). The incidence of prostate cancer in Korea increased by $12.8 \%$ annually between 1999 and 2010 (2). Testing for prostate-specific antigen (PSA) has led to increased diagnoses of localized prostate cancer; however, despite this downward stage migration, a number of patients are still diagnosed with metastatic prostate cancer (2). According to the Korea Central Cancer Registry, between 2006 and 2010 $273.3 \%$ of patients with prostate cancer were diagnosed with loco-regional disease, whereas $9.0 \%$ presented with distant disease (extension to organs other than the prostate, or metastases to distant lymph nodes or organs) (2).

Androgen deprivation therapy (ADT), including bilateral orchiectomy and treatment with luteinizing hormone-releasing hormone (LHRH) agonists with or without anti-androgens, is a well-established initial treatment for metastatic prostate cancer (3). Despite the initial positive response, a number of patients may experience significant adverse effects (4-6). Castration-resistant prostate cancer (CRPC) is inevitable following a variable period of response to ADT (7-10). Strategies to delay CRPC and minimize the adverse effects of ADT, including intermittent androgen deprivation therapy (IADT) or novel agents, remains an active area of investigation (11). 
Table I. Baseline characteristics of patients who received continuing MAB, and those who converted to monotherapy once the PSA nadir was reached following initial MAB.

\begin{tabular}{lcc}
\hline Characteristics & $\begin{array}{c}\text { Continuing MAB } \\
(\mathrm{n}=227)\end{array}$ & $\begin{array}{c}\text { Conversion to } \\
\text { monotherapy }(\mathrm{n}=66)\end{array}$ \\
\hline Median age \pm SD & $68.74 \pm 7.48$ & $70.94 \pm 7.60$ \\
LN pretreatment PSA & $6.41 \pm 1.42$ & $5.78 \pm 1.29$ \\
Gleason score & & 0.906 \\
$\leq 6$ & $6(2.6 \%)$ & $5(7.6 \%)$ \\
7 & $17(7.5 \%)$ & $4(6.0 \%)$ \\
$\geq 8$ & $204(89.9 \%)$ & $57(8.64 \%)$ \\
Pretreatment extent of bone metastasis & & $17(25.8 \%)$ \\
Focal & $53(23.3 \%)$ & $40(60.6 \%)$ \\
Multiple & $104(45.8 \%)$ & $9(13.6 \%)$ \\
Disseminated & $70(30.9 \%)$ & $0.018^{\mathrm{a}}$ \\
\hline
\end{tabular}

${ }^{a} \mathrm{P}<0.05$ indicates a statistically significant difference. MAB, maximal androgen blockade; SD, standard deviation; LN, natural logarithm; PSA, prostate-specific antigen.

During the management of advanced prostate cancer, previous studies have demonstrated that IADT is at least as effective as continuous treatment with regard to disease progression and overall survival (12-14). However, IADT should be considered as an alternative therapeutic approach to CADT, accounting for the caveats and cautions suggested by meta-analysis of eight randomized controlled trials of intermittent vs. continuous ADT (12-14).

In high-volume metastatic prostatic cancer, including various lymph node or bone metastases, initial bulky tumors and high baseline PSA levels, IADT is not a suitable treatment modality (15-17). In these patients, continuous ADT must remain the standard treatment approach (14). Continuous ADT comprises surgical castration, LHRH agonist with/without anti-androgen and LHRH antagonist (14).

Therefore, the present study compared the efficacy of continuous maximal androgen blockade (MAB) and conversion to monotherapy, with an LHRH agonist or orchiectomy, after reaching PSA nadir following initial MAB therapy in patients with metastatic prostate cancer at initial presentation. Additionally, various factors affecting disease progression were also investigated.

\section{Materials and methods}

A retrospective review of the medical records of male patients diagnosed with prostate cancer with bone metastasis between 1996 and 2011 at the Asan Medical Center (Seoul, Korea) was performed. A total of 354 patients diagnosed with prostate cancer with bone metastasis received MAB as a first-line therapy. Patients with no recorded follow up for $\geq 1$ year, or who had incomplete data, were excluded, and the remaining 293 patients formed the study cohort. Bone metastasis was assessed using a radionuclide bone scan. The median age at diagnosis was 70.2 years (range, $47-89$ years).

MAB consisted of an oral anti-androgen $(750 \mathrm{mg} / \mathrm{day}$ flutamide or $50 \mathrm{mg} /$ day bicalutamide) with orchiectomy
(89/293,30.4\%) or an LHRH agonist (3.75 mg/month leuprolide or $3.6 \mathrm{mg} / \mathrm{month}$ goserelin acetate; 204/293, 69.6\%). Following attainment of the PSA nadir with initial MAB treatment (at a median of 8.5 months), patients received maintenance therapy with MAB (227/293, 77.5\%), monotherapy (66/293, 22.5\%) with an LHRH agonist or no treatment following bilateral orchiectomy.

PSA was recorded every 3 months, and a bone scan was performed every 6-12 months. However, if clinical progression was suspected due to the elevation of PSA levels at two consecutive readings or the development of skeletal symptoms, an immediate image study, including an X-ray, computed tomography or bone scan, was conducted.

The efficacy of ADT was assessed via serum PSA levels and bone lesion responses (18). Disease progression was defined as progression to CRPC (biochemical progression and/or radiological progression). CRPC was defined by using the Prostate Cancer Working Group criteria as a continuous rise in PSA levels (biochemical progression and/or a radiological progression) even when the serum testosterone level remained in the castrate range $(<50 \mathrm{ng} / \mathrm{dl})$ due to surgical orchiectomy or medical therapy (18). Biochemical progression was defined as three consecutive rises in PSA levels one week apart, resulting in two $50 \%$ increases over the nadir with PSA concentrations of $>2 \mathrm{ng} / \mathrm{ml}$. Radiological progression was defined as the appearance of $\geq 2$ novel lesions on a bone scan.

The propensity score was determined from a non-parsimonious logistic regression model for treatment with continuous MAB vs. conversion to monotherapy. The variables included in this model were age, Gleason score, pretreatment PSA and pretreatment extent of bone metastasis. Patients receiving continuous MAB were matched at a ratio of 2:1 (105:53) with patients converting to monotherapy, by using the closest available pair matching method (19). Model discrimination was assessed with c-statistics $(\mathrm{P}=0.724)$ and model calibration was assessed with Hosmer-Lemeshow statistics $\left(\chi^{2}=4.662\right.$; $\mathrm{P}=0.793$ ). 
Table II. Baseline characteristics of propensity-matched patients.

\begin{tabular}{lccr}
\hline Characteristics & $\begin{array}{c}\text { Continuing } \\
\text { MAB (n=102) }\end{array}$ & $\begin{array}{c}\text { Conversion to } \\
\text { monotherapy (n=53) }\end{array}$ & P-value \\
\hline Median age \pm SD & $69.38 \pm 8.16$ & $70.38 \pm 8.50$ & 0.068 \\
LN pretreatment PSA & $4.82 \pm 1.48$ & $4.98 \pm 1.21$ & 0.683 \\
Gleason score & & & 0.495 \\
$\leq 6$ & $2(2.0 \%)$ & $1(1.9 \%)$ & \\
7 & $4(3.9 \%)$ & $1(1.9 \%)$ & 0.942 \\
$\geq 8$ & $96(94.1 \%)$ & $51(96.2 \%)$ & \\
Pretreatment extent of bone metastasis & & $12(22.6 \%)$ & \\
Focal & $24(23.5 \%)$ & $34(64.2 \%)$ & $7(13.2 \%)$ \\
Multiple & $63(61.8 \%)$ & & \\
Disseminated & $17(14.7 \%)$ & & \\
\hline
\end{tabular}

MAB, maximal androgen blockade; LN, natural logarithm; PSA, prostate-specific antigen.

A

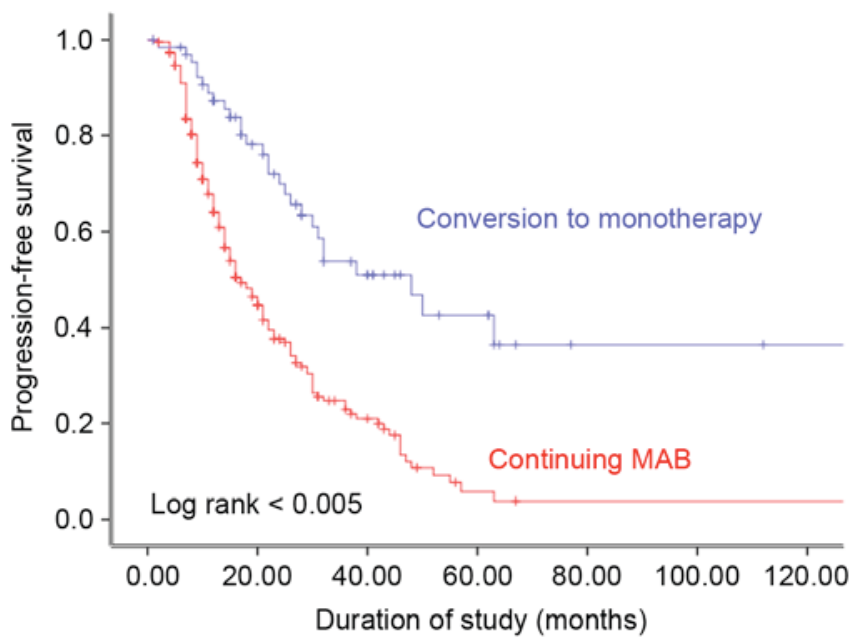

B

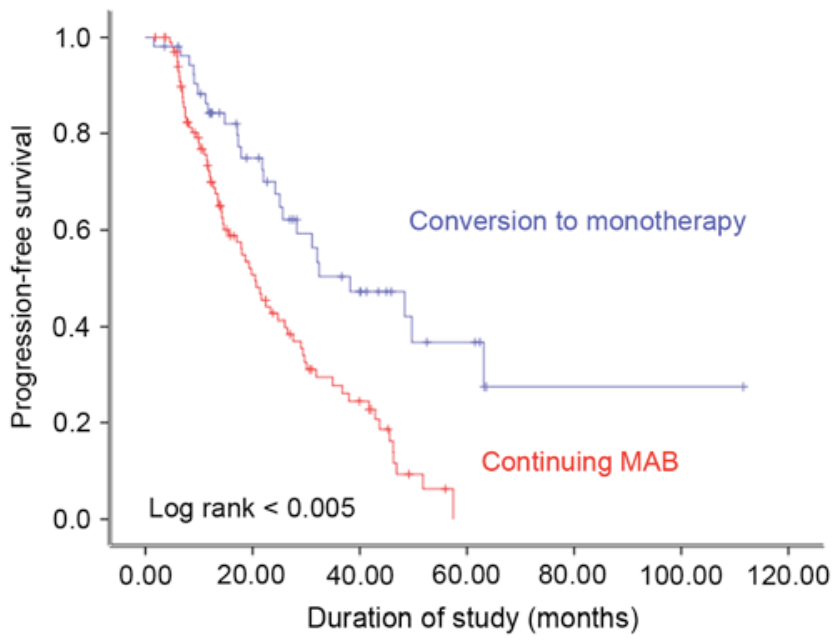

Figure 1. Kaplan-Meier determination of progression-free survival time according to type of maintenance androgen deprivation therapy for (A) all patients without adjustment $(n=293 ; \log$ rank $<0.005)$ and $(B)$ propensity score matched pairs $(n=155 ; \log$ rank $<0.005)$. MAB, maximal androgen blockade.

To evaluate disease progression and compare treatment methods, $\chi^{2}$ and Student's $t$-tests were used as appropriate. Survival was estimated using the Kaplan-Meier method and compared using the log-rank test. A Cox proportional hazards model was used for multivariate analysis. All statistical analyses were performed with SPSS 18.0 software (SPSS Inc., Chicago, IL, USA). $\mathrm{P}<0.05$ was considered to indicate a statistically significant difference.

\section{Results}

The median follow-up period was 39.1 months (range, 12-170 months). Following initial MAB treatment, serum PSA had decreased to an undetectable level in $37.4 \%$ of patients, and to a detectable nadir in $62.6 \%$. The median time to a PSA nadir was 8.5 months; following this nadir, $77.5 \%$ of the patients stayed on MAB and the remaining $22.5 \%$ were switched to monotherapy.
The clinical characteristics of the 293 patients based on post-nadir treatment status are presented in Table I. Patients on maintenance monotherapy had more favorable clinicopathological characteristics, including a lower pretreatment PSA level $(\mathrm{P}=0.005)$ and a lower metastatic burden $(\mathrm{P}=0.018)$. Following propensity score matching, the baseline clinicopathological characteristics remained similar between the groups (Table II).

After a median treatment period of 22.7 months, 110 (70.9\%) patients exhibited evidence of progression to CRPC, of which $95(86.4 \%)$ demonstrated biochemical progression and $15(13.6 \%)$ had radiological progression. Kaplan-Meier analysis demonstrated that progression-free survival (PFS) was significantly associated with the type of maintenance ADT administered to the patients, prior to and following propensity score matching (log rank, $\mathrm{P}<0.005$; Fig. 1). The median PFS was 19.5 and 28.8 months following continuous $\mathrm{MAB}$ and conversion to monotherapy, respectively $(\mathrm{P}=0.008)$. 
Table III. Cox proportional hazards model multivariate analysis of predictive factors of progression-free survival.

\begin{tabular}{lcc}
\hline Characteristics & Hazard ratio & $95 \%$ CI \\
\hline ADT method & & \\
Continuing MAB & 2.293 & $1.536-3.424$ \\
Conversion to monotherapy & 1 & $0.975-1.009$ \\
Age & 0.992 & $<0.001^{\mathrm{a}}$ \\
Gleason score & & 0.364 \\
$\leq 6$ & 1 & $0.600-7.279$ \\
7 & 2.090 & $0.663-6.720$ \\
$\geq 8$ & 2.110 & $0.908-1.095$ \\
LN pretreatment PSA & 0.997 & 0.450 \\
Pretreatment extent of bone metastasis & & 0.206 \\
Focal & 1 & 0.950 \\
Multiple & 1.666 & $1.090-2.524$ \\
Disseminated & 2.547 & $1.614-4.022$ \\
\hline
\end{tabular}

${ }^{\mathrm{a}} \mathrm{P}<0.05$ indicates a statistically significant difference. ADT, androgen deprivation therapy; MAB, maximal androgen blockade; LN, natural logarithm; PSA, prostate-specific antigen.

Multivariate analysis using the Cox proportional hazard model revealed that the type of maintenance ADT and the pretreatment extent of bone metastasis were independent predictive factors of disease progression (Table III).

\section{Discussion}

Even with the availability of PSA screening and the resulting reduction in cancer-stage progression, numerous patients with prostate cancer are initially diagnosed with metastatic disease, with $2.6 \%$ of patients in the CaPSURE ${ }^{\mathrm{TM}}$ trial possessing metastatic disease at the time of diagnosis (20). Of the patients in the present study, $16.4 \%$ were initially diagnosed with metastatic prostate cancer. Among these patients, ADT was the primary treatment for metastatic disease, with its use increasing throughout the study period.

Advanced prostate cancer typically becomes androgenindependent following castration (8). The duration of response to ADT in metastatic prostate cancer is $~ 14-20$ months (7-10). To maximize the effect of ADT, numerous strategies were used for the treatment of patients (continuous ADT vs. intermittent ADT; MAB vs. mono-ADT) (21).

As aforementioned, the relative efficacy of IADT has yet to be established in metastatic prostate cancer. Previous studies have demonstrated that IADT was associated with a poorer prognosis and PFS for patients with metastatic prostate cancer $(8,20,22)$. In the Southwest Oncology Group (SWOG) 9,346 trial, the median overall survival exhibited an absolute difference moderately $>6$ months in favor of continuous therapy; 5.1 years in the intermittent-therapy group compared with 5.8 years in the continuous-therapy group (22).

Therefore, continuous ADT remains the standard treatment for metastatic prostate cancer; however, which continuous ADT methods (MAB vs. mono-ADT) are beneficial in metastatic settings remain to be established. Systematic reviews have demonstrated that MAB using non-steroidal anti-androgens appears to yield a small survival advantage $(<5 \%)$ beyond five years, as compared with mono-ADT (surgical castration or LHRH agonists) (23-25).

The European Association of Urology and The European Society of Medical Oncology recommend short-term administration of anti-androgen for between 1 and 4 weeks only to minimize the risk of the 'flare-up' phenomenon in patients with advanced metastatic prostate cancer $(26,27)$. In the present study, maintenance of the anti-androgen treatment was required until the PSA nadir was reached at $\sim 8.5$ months.

In the present study, conversion to monotherapy prolonged PFS for 9.3 months, as compared with continuing MAB. In addition, a marked difference was observed in PFS in patients with metastatic prostate cancer who received ADT. Patients who were treated with a continuous LHRH agonist, or observation following orchiectomy had improved PFS, as compared with those who received continuous MAB following attainment of the PSA nadir (28.8 vs. 19.5 months). Due to variations in the baseline characteristics, including pretreatment PSA and the extent of bone metastasis, propensity score matched analysis was performed. Prior to and following matching, conversion to monotherapy was observed to be superior to continuous MAB with respect to PFS in patients with hormone-sensitive prostate cancer. Continuous MAB was associated with a 2.3-fold higher risk of prostate cancer progression, compared with conversion to monotherapy.

The inappropriate use of hormonal therapy, including short-term, intermittent, incomplete ADT or anti-androgen only, may lead to disease progression and an increased proportion of cancer cells with a higher malignant potential (28). A patient's progression-free survival may be affected by a delay in the appearance of androgen-insensitive clones, following conversion to monotherapy. It is hypothesized that continuous MAB treatment may have facilitated the earlier development of androgen-insensitive clones; by contrast, conversion to monotherapy may have delayed the development 
of androgen-insensitive clones. MAB is associated with treatment-associated costs and increased adverse effects, including increased risk of cardiovascular disease, diabetes and metabolic abnormalities (29). Therefore, maintenance therapy with an LHRH agonist, or observation following orchiectomy, may be associated with a reduction in adverse effects and treatment-associated costs, in addition to yielding improved PFS, as compared with the continuation of MAB therapy.

One limitation of retrospective studies is the potential for selection bias. To mitigate this possibility during the present study, propensity score matching analysis was performed. Furthermore, the quality of life associated with specific treatments was not evaluated, and an investigation of treatment-associated adverse events was not performed. Due to the intermediate duration of follow-up (39.1 months), the impact of ADT on cancer-specific or overall survival was not analyzed in the present study.

In conclusion, conversion to monotherapy following attainment of the PSA nadir with initial MAB therapy prolonged progression-free survival in patients with prostate cancer. The results suggest that monotherapy maintenance following initial MAB therapy may benefit patients by reducing the incidence of side effects without decreasing treatment efficacy.

\section{References}

1. Jemal A, Siegel R, Ward E, Hao Y, Xu J, Murray T and Thun MJ: Cancer statistics, 2008. CA Cancer J Clin 58: 71-96, 2008.

2. Jung KW, Won YJ, Kong HJ, Oh CM, Shin A and Lee JS: Survival of Korean adult cancer patients by stage at diagnosis, 2006-2010: National cancer registry study. Cancer Res Treat 45: $162-171,2013$.

3. Higgins JP, Altman DG, Gøtzsche PC, Jüni P, Moher D, Oxman AD, Savovic J, Schulz KF, Weeks L, Sterne JA, et al: The Cochrane Collaboration's tool for assessing risk of bias in randomised trials. BMJ 343: d5928, 2011.

4. Salonen AJ, Taari K, Ala-Opas M, Viitanen J, Lundstedt S and Tammela TL; FinnProstate Group: The FinnProstate study VII: intermittent versus continuous androgen deprivation in patients with advanced prostate cancer. J Urol 187: 2074-2081, 2012.

5. Taylor LG, Canfield SE and Du XL: Review of major adverse effects of androgen-deprivation therapy in men with prostate cancer. Cancer 115: 2388-2399, 2009.

6. Schwandt A and Garcia JA: Complications of androgen deprivation therapy in prostate cancer. Curr Opin Urol 19: 322-326, 2009.

7. Crawford ED, Eisenberger MA, McLeod DG, Spaulding JT, Benson R, Dorr FA, Blumenstein BA, Davis MA and Goodman PJ: A controlled trial of leuprolide with and without flutamide in prostatic carcinoma. N Engl J Med 321: 419-424, 1989.

8. Sharifi N, Gulley JL and Dahut WL: Androgen deprivation therapy for prostate cancer. JAMA 294: 238-244, 2005.

9. Mottet N, Van Damme J, Loulidi S, Russel C, Leitenberger A and Wolff JM; TAP22 Investigators Group: Intermittent hormonal therapy in the treatment of metastatic prostate cancer: A randomized trial. BJU Int 110: 1262-1269, 2012.

10. Seidenfeld J, Samson DJ, Hasselblad V, Aronson N, Albertsen PC, Bennett CL and Wilt TJ: Single-therapy androgen suppression in men with advanced prostate cancer: A systematic review and meta-analysis. Ann Intern Med 132: 566-577, 2000.

11. Alva AS and Hussain M: Initial management of metastatic prostate cancer. In: Comprehensive Textbook of Genitourinary Oncology. Scardino PT, Linehan WM, Zelefsky MJ and Vogelzang NJ (eds). Lippincott Williams \& Wilkins, Philadelphia, pp251-261, 2011.

12. Calais da Silva F, Calais da Silva FM, Gonçalves F, Santos A, Kliment J, Whelan P, Oliver T, Antoniou N, Pastidis S, Marques Queimadelos A and Robertson C: Locally advanced and metastatic prostate cancer treated with intermittent androgen monotherapy or maximal androgen blockade: Results from a randomised phase 3 study by the South European Uroncological Group. Eur Urol 66: 232-239, 2014.
13. Calais da Silva FE, Bono AV, Whelan P, Brausi M, Marques Queimadelos A, Martin JA, Kirkali Z, Calais da Silva FM and Robertson C: Intermittent androgen deprivation for locally advanced and metastatic prostate cancer: Results from a randomised phase 3 study of the South European Uroncological Group. Eur Urol 55: 1269-1277, 2009.

14. Tsai HT, Penson DF, Makambi KH, Lynch JH, Van Den Eeden SK and Potosky AL: Efficacy of intermittent androgen deprivation therapy vs conventional continuous androgen deprivation therapy for advanced prostate cancer: A meta-analysis. Urology 82: 327-333, 2013.

15. Prapotnich D, Fizazi K, Escudier B, Mombet A, Cathala N and Vallancien G: A 10-year clinical experience with intermittent hormonal therapy for prostate cancer. Eur Urol 43: 233-240, 2003.

16. Salonen AJ, Viitanen J, Lundstedt S, Ala-Opas M, Taari K and Tammela TL; FinnProstate Group: Finnish multicenter study comparing intermittent to continuous androgen deprivation for advanced prostate cancer: Interim analysis of prognostic markers affecting initial response to androgen deprivation. J Urol 180: 915-920, 2008

17. Grossfeld GD, Small EJ and Carroll PR: Intermittent androgen deprivation for clinically localized prostate cancer: Initial experience. Urology 51: 137-144, 1998.

18. Scher HI, Halabi S, Tannock I, Morris M, Sternberg CN, Carducci MA, Eisenberger MA, Higano C, Bubley GJ, Dreicer R, et al: Design and end points of clinical trials for patients with progressive prostate cancer and castrate levels of testosterone: Recommendations of the Prostate Cancer Clinical Trials Working Group. J Clin Oncol 26: 1148-1159, 2008.

19. D'Agostino RB Jr: Propensity score methods for bias reduction in the comparison of a treatment to a non-randomized control group. Stat Med 17: 2265-2281, 1998.

20. Ryan CJ, Elkin EP, Small EJ, Duchane J and Carroll P: Reduced incidence of bony metastasis at initial prostate cancer diagnosis: Data from CaPSURE. Urol Oncol 24: 396-402, 2006.

21. Shiota M and Eto M: Current status of primary pharmacotherapy and future perspectives toward upfront therapy for metastatic hormone-sensitive prostate cancer. Int J Urol 23: 360-369, 2016.

22. Hussain M, Tangen CM, Berry DL, Higano CS, Crawford ED Liu G, Wilding G, Prescott S, Kanaga Sundaram S, Small EJ, et al: Intermittent versus continuous androgen deprivation in prostate cancer. N Engl J Med 368: 1314-1325, 2013.

23. Maximum androgen blockade in advanced prostate cancer: An overview of the randomised trials with 3283 deaths in 5710 patients. Prostate Cancer Trialists' Collaborative Group. Lancet 346: 265-269, 1995.

24. Akaza H, Hinotsu S, Usami M, Arai Y, Kanetake H, Naito S and Hirao Y; Study Group for the Combined Androgen Blockade Therapy of Prostate Cancer: Combined androgen blockade with bicalutamide for advanced prostate cancer: Long-term follow-up of a phase 3, double-blind, randomized study for survival. Cancer 115: 3437-3445, 2009.

25. Schmitt B, Bennett C, Seidenfeld J, Samson D and Wilt T: Maximal androgen blockade for advanced prostate cancer. Cochrane Database Syst Rev: CD001526, 2000.

26. Heidenreich A, Bastian PJ, Bellmunt J, Bolla M, Joniau S, van der Kwast T, Mason M, Matveev V, Wiegel T, Zattoni F, et al: EAU guidelines on prostate cancer. Part II: Treatment of advanced, relapsing and castration-resistant prostate cancer. Eur Urol 65: 467-479, 2014

27. Horwich A, Parker C, de Reijke T and Kataja V, ESMO Guidelines Working Group: Prostate cancer: ESMO clinical practice guidelines for diagnosis, treatment and follow-up. Ann Oncol 24 (Suppl 6): vi106-vi114, 2013.

28. Akaza H, Labrie F and Namiki M: A way of thinking of a MAB therapy for local/locally advanced prostate cancer: The theory and recent evaluation. Gan To Kagaku Ryoho 34: 657-669, 2007 (In Japanese)

29. Sharifi N, Gulley JL and Dahut WL: An update on androgen deprivation therapy for prostate cancer. Endocr Relat Cancer 17: R305-R315, 2010. 